JOURNAL OF

SYMPLECTIC GEOMETRY

Volume 9, Number 3, 403-428, 2011

\title{
ON COHOMOLOGICAL DECOMPOSITION OF ALMOST-COMPLEX MANIFOLDS AND DEFORMATIONS
}

\author{
Daniele Angella And Adriano Tomassini
}

\begin{abstract}
While small deformations of compact Kähler manifolds are Kähler too, we prove that the cohomological property to be $\mathcal{C}^{\infty}$-pure-and-full is not a stable condition under small deformations. This property, which has been recently introduced and studied by $\mathrm{Li}$ and Zhang in [24] and Draghici et al. in $[\mathbf{1 3}, \mathbf{1 4}]$, is weaker than the Kähler one and characterizes the almost-complex structures inducing a decomposition in cohomology. We also study the stability of this property along curves of almost-complex structures constructed starting from the harmonic representatives in special cohomology classes.
\end{abstract}

\section{Introduction}

Let $(M, J)$ be a compact almost-complex $2 n$-dimensional manifold and let $\omega$ be a symplectic form on $M$. Then $J$ is said to be $\omega$-tamed if $\omega(\cdot, J \cdot)>0$ and $\omega$-compatible (or $\omega$-calibrated) if $g(\cdot, \cdot):=\omega(\cdot, J \cdot)$ is a $J$-Hermitian metric. Define the tamed cone $\mathcal{K}_{J}^{\mathrm{t}}$ as the open convex cone given by the projection in cohomology of the space of the symplectic forms taming $J$, namely

$$
\mathcal{K}_{J}^{\mathrm{t}} \stackrel{\text { def }}{=}\left\{[\omega] \in H_{d R}^{2}(M ; \mathbb{R}) \mid J \text { is } \omega \text {-tamed }\right\},
$$

and the compatible cone $\mathcal{K}_{J}^{c}$ as its subcone given by the projection of the space of the symplectic forms compatible with $J$, namely

$$
\mathcal{K}_{J}^{\mathrm{c}} \stackrel{\text { def }}{=}\left\{[\omega] \in H_{d R}^{2}(M ; \mathbb{R}) \mid J \text { is } \omega \text { - compatible }\right\} .
$$

$\mathrm{Li}$ and Zhang proved in [24, Corollay 3.2] that if $J$ is integrable and $\mathcal{K}_{J}^{\mathrm{c}}$ is non-empty then the following relation between the two cones holds:

$$
\mathcal{K}_{J}^{\mathrm{t}}=\mathcal{K}_{J}^{\mathrm{c}}+\left(\left(H_{\bar{\partial}}^{2,0}(M) \oplus H_{\bar{\partial}}^{0,2}(M)\right) \cap H_{d R}^{2}(M ; \mathbb{R})\right) ;
$$

they also proved (see [24, Theorem 1.2]) that, given a complex compact surface $(M, J)$, if there is a symplectic structure $\omega$ such that $J$ is $\omega$-tamed then $(M, J)$ admits a Kähler structure (see also [29, Proposition 1.6]), i.e. 
in such a case $\mathcal{K}_{J}^{\mathrm{t}}$ is empty if and only if $\mathcal{K}_{J}^{\mathrm{c}}$ is empty: this gives a partial answer to a question of Donaldson's, [12, Question 2]. Therefore, the problem of finding explicit examples of compact complex non-Kähler manifolds, admitting a holomorphic structure tamed by a symplectic form, makes sense only in dimension higher than 4, as asked by Li and Zhang in [24, p. 678] and Streets and Tian [29, Question 1.7].

In view of $[\mathbf{6}$, Theorem $\mathrm{A}]$, the most natural category in which one can find non-Kähler manifolds is that one of nilmanifolds: we prove that no such example could be found among the nilmanifolds of dimension 6 (see Theorem 3.4).

In order to generalize (1.1) for an arbitrary almost-complex structure, $\mathrm{Li}$ and Zhang introduced in $[\mathbf{2 4}]$ the concept of $\mathcal{C}^{\infty}$-pure-and-full almostcomplex structure. More precisely, an almost-complex structure $J$ is said to be $\mathcal{C}^{\infty}$-pure-and-full if it induces the decomposition

$$
H_{d R}^{2}(M ; \mathbb{R})=H_{J}^{(1,1)}(M)_{\mathbb{R}} \oplus H_{J}^{(2,0),(0,2)}(M)_{\mathbb{R}},
$$

where the group $H_{J}^{(2,0),(0,2)}(M)_{\mathbb{R}}$ (respectively, $\left.H_{J}^{(1,1)}(M)_{\mathbb{R}}\right)$ is given by the projection in cohomology of the space $\left(\wedge^{2,0} M \oplus \wedge^{0,2} M\right) \cap \wedge^{2} M$ (respectively, $\left.\wedge^{1,1} M \cap \wedge^{2} M\right)$; more in general, $J$ is said to be $\mathcal{C}^{\infty}$-full if the equality

$$
H_{d R}^{2}(M ; \mathbb{R})=H_{J}^{(1,1)}(M)_{\mathbb{R}}+H_{J}^{(2,0),(0,2)}(M)_{\mathbb{R}}
$$

holds, namely if there exists a basis of $H_{d R}^{2}(M ; \mathbb{R})$ formed by classes having at least one type of pure degree representative.

In $\left[\mathbf{2 4}\right.$, Theorem 1.1], Li and Zhang proved that if $J$ is $\mathcal{C}^{\infty}$-full and if $\mathcal{K}_{J}^{\mathrm{c}}$ is non-empty, then

$$
\mathcal{K}_{J}^{\mathrm{t}}=\mathcal{K}_{J}^{\mathrm{c}}+H_{J}^{(2,0),(0,2)}(M)_{\mathbb{R}}
$$

where $H_{J}^{(2,0),(0,2)}(M)_{\mathbb{R}}$ generalizes the group

$$
\left(H_{\bar{\partial}}^{2,0}(M) \oplus H_{\bar{\partial}}^{0,2}(M)\right) \cap H_{d R}^{2}(M ; \mathbb{R})
$$

in (1.1).

In [24] dual notions starting from the space of currents are also defined: we will recall in Section 2 what a pure-and-full almost-complex structure is. Further studies about $\mathcal{C}^{\infty}$-pure-and-full almost-complex structures have been carried out in $[\mathbf{1 3}, \mathbf{2 0}]$.

In particular, Draghici et al. proved in [13, Theorem 2.3] that every almost-complex structure on a compact four-dimensional manifold is $\mathcal{C}^{\infty}$-pure-and-full.

As a consequence of the last two quoted results, (see [24, Corollary $1.1])$, if $(M, J)$ is a compact almost complex four-manifold such that $\mathcal{K}_{J}^{\mathrm{c}}$ 
is non-empty, then

$$
\mathcal{K}_{J}^{\mathrm{t}}=\mathcal{K}_{J}^{\mathrm{c}}+H_{J}^{(2,0),(0,2)}(M)_{\mathbb{R}}
$$

In particular, if $b^{+}(M)=\operatorname{dim}_{\mathbb{R}} H_{J}^{(1,1)}(M)_{\mathbb{R}}=1$, then $\mathcal{K}_{J}^{\mathrm{t}}=\mathcal{K}_{J}^{\mathrm{c}}$.

In real dimension greater than 4 , things are different. Indeed, for example, there are almost-complex structures on compact six-dimensional solvmanifolds which are not $\mathcal{C}^{\infty}$-pure (see [20, Example 3.3]). This turns our attention to the six-dimensional case.

In this paper, we are interested in studying small deformations of $\mathcal{C}^{\infty}$-pure-and-full complex structures. The celebrated theorem of Kodaira and Spencer, [22, Theorem 15], states that Kähler metrics on compact complex manifolds are stable under small deformations; Alessandrini and Bassanelli proved in $[\mathbf{2}]$ that this stability fails to be true for the class of $p$-Kähler manifolds, where $p \in\{2, \ldots, n-1\}$ (see [1] for the precise definition) e.g., for the class of balanced metrics, namely the $J$-Hermitian metrics on compact complex manifolds whose fundamental form $\omega$ satisfies $d \omega^{n-1}=0$.

Since the $\mathcal{C}^{\infty}$-pure-and-full condition is weaker than the Kähler one (more precisely, as a consequence of [24], see Theorem 2.4, every compact complex manifold verifying the $\partial \bar{\partial}$-Lemma is $\mathcal{C}^{\infty}$-pure-and-full), it could be interesting to establish if the $\mathcal{C}^{\infty}$-pure-and-full complex structures are stable under small deformations. As hinted by Li and Zhang in a previous version of [24], we study the stability of the standard complex structure on the Iwasawa manifold and try to deform a $\mathcal{C}^{\infty}$-pure-and-full almost-complex structure starting with $J$-anti-invariant forms as explained in [23].

In [26], Nakamura computed the small deformations of the Iwasawa manifold $X$, dividing them in three classes. Then, a direct computation shows that the complex structure on $X$ is $\mathcal{C}^{\infty}$-pure-and-full.

We prove that (see Theorem 3.1 for the precise statement) the small deformations of class (i) are $\mathcal{C}^{\infty}$-pure-and-full while those ones of classes (ii) and (iii) are not. Hence, as a corollary we get the following (see Section 3).

Theorem 3.2. Compact complex $\mathcal{C}^{\infty}$-pure-and-full (or $\mathcal{C}^{\infty}$-pure or $\mathcal{C}^{\infty}$-full or pure-and-full or pure or full) manifolds are not stable under small deformations of the complex structure.

Furthermore, we show the following:

Theorem 3.6. Let $X:=(\mathbb{Z}[\mathrm{i}])^{3} \backslash\left(\mathbb{C}^{3}, *\right)$ be the Iwasawa manifold. Then any small complex deformation of $X$ cannot be tamed by any symplectic form.

As $\mathcal{C}^{\infty}$-pure-and-full property is defined for an arbitrary almost-complex structure (even not integrable), we study its stability along curves of almostcomplex structures $\left\{J_{t}\right\}_{t \in(-\varepsilon, \varepsilon)}$, too.

In [14], it is proved the semi-continuity property of $h_{J}^{ \pm}$for an almostcomplex structure on a compact four-dimensional manifold. More precisely, 
if $M$ is a compact four-manifold with an almost-complex structure $J$ such that $\mathcal{K}_{J}^{\mathrm{c}} \neq \emptyset$, then for any almost complex structure $J^{\prime}$ in a sufficiently small neighborhood of $J$ the following holds:

- $\mathcal{K}_{J^{\prime}}^{c} \neq \emptyset$,

- $h_{J}^{+}(M) \leq h_{J^{\prime}}^{+}(M)$,

- $h_{J}^{-}(M) \geq h_{J^{\prime}}^{-}(M)$.

In [23], curves of almost-complex structures parametrized by real forms of pure degree $(2,0)+(0,2)$ are constructed. Using this construction, we prove that (see Theorem 4.1 for the precise statement) there exists a family $\left\{N^{6}(c)\right\}_{c}$ of compact cohomologically Kähler manifolds with no Kähler metrics such that

(i) $N^{6}(c)$ admits a $\mathcal{C}^{\infty}$-pure-and-full almost-complex structure $J$,

(ii) each harmonic form of type $(2,0)+(0,2)$ gives rise to a curve $\left\{J_{t}\right\}_{t \in(-\varepsilon, \varepsilon)}$ of $\mathcal{C}^{\infty}$-pure-and-full almost-complex structures on $N^{6}(c)$,

(iii) furthermore, the map

$$
t \mapsto \operatorname{dim}_{\mathbb{R}} H_{J_{t}}^{(2,0),(0,2)}\left(N^{6}(c)\right)_{\mathbb{R}}
$$

is an upper-semicontinuous function at $t=0$.

In particular, we get the upper-semicontinuity property of $h_{J}^{-}$for this sixdimensional example.

We recall that, for a suitable $c \in \mathbb{R}$, the completely solvable Lie group

$$
\operatorname{Sol}(3) \stackrel{\text { def }}{=}\left\{\left(\begin{array}{cccc}
\mathrm{e}^{c z} & 0 & 0 & x \\
0 & \mathrm{e}^{-c z} & 0 & y \\
0 & 0 & 1 & z \\
0 & 0 & 0 & 1
\end{array}\right) \in \mathrm{GL}(4 ; \mathbb{R}) \mid x, y, z \in \mathbb{R}\right\}
$$

admits a cocompact discrete subgroup $\Gamma(c)$; we define

$$
M \stackrel{\text { def }}{=} \Gamma(c) \backslash \operatorname{Sol}(3)
$$

and

$$
N^{6}(c) \stackrel{\text { def }}{=} M \times M
$$

the manifold $N^{6}(c)$ first appeared in [7] as an example of a cohomologically Kähler manifold; Fernández et al. proved in [17] that it has no Kähler structures.

In Section 2, we fix the notation, recall the main results on $\mathcal{C}^{\infty}$-pureand-full almost-complex structures from $[\mathbf{2 4}, \mathbf{1 3}]$ and $[\mathbf{2 0}]$ and we give an example of six-dimensional (compact) non-Kähler solvmanifold endowed with a $\mathcal{C}^{\infty}$-pure-and-full and pure-and-full almost complex structure. In Section 3, we prove the instability Theorem 3.2. Then, as a consequence of a general fact (see Theorem 3.3) true for six-dimensional nilmanifolds (i.e. compact quotient of nilpotent simply connected Lie groups by uniform dicrete subgroups), we show that the deformed complex structures 
described in Theorem 3.1 cannot be tamed by any symplectic form $\omega$ on the Iwasawa manifold. Finally, in Section 4, we recall how a cohomology class in $H_{J}^{(2,0),(0,2)}(M)_{\mathbb{R}}$ gives rise to a curve of almost-complex structures on $(M, J)$; we provide several examples of curves of $\mathcal{C}^{\infty}$-pure-and-full almost-complex structures on four and six-dimensional compact manifolds.

\section{2. $\mathcal{C}^{\infty}$-pure-and-full almost-complex structures}

Let $(M, J)$ be an almost-complex compact $2 n$-manifold. The endomorphism $J$ on $T M \otimes \mathbb{C}$, having eigenvalues $\mathrm{i}$ and $-\mathrm{i}$, induces a decomposition of $\wedge^{\bullet}(M ; \mathbb{C})$ through $\wedge_{J}^{p, q} M:=: \wedge^{p, q} M$, namely $\wedge^{k} M=\bigoplus_{p+q=k} \wedge_{J}^{p, q} M$. We ask about when this decomposition holds in cohomology. Define $H_{J}^{(p, q)}(M)$ as the projection in de Rham cohomology of the space $\wedge^{p, q} M$; define $H_{J}^{(p, q),(q, p)}(M)_{\mathbb{R}}$ as the projection in de Rham cohomology of the space $\left(\wedge^{p, q} M \oplus \wedge^{q, p} M\right) \cap \wedge^{p+q} M$. (As a matter of notation, bigraduation without further specification refers to complex forms, single graduation to real ones). In other words:

$$
H_{J}^{(p, q),(q, p)}(M)_{\mathbb{R}}=\left\{[\alpha] \in H_{d R}^{p+q}(M ; \mathbb{R}) \mid \alpha \in\left(\wedge_{J}^{p, q} M \oplus \wedge_{J}^{q, p} M\right) \cap \wedge^{p+q} M\right\} .
$$

If $S$ is a set of pairs $(p, q)$, we define likewise

$$
H_{J}^{S}(M) \stackrel{\text { def }}{=}\left\{[\alpha] \in H_{d R}^{\bullet}(M ; \mathbb{C}) \mid \alpha \in \bigoplus_{(p, q) \in S} \wedge^{p, q} M\right\}
$$

and

$$
H_{J}^{S}(M)_{\mathbb{R}} \stackrel{\text { def }}{=} H_{J}^{S}(M) \cap H_{d R}^{\bullet}(M ; \mathbb{R}) .
$$

$\mathrm{Li}$ and Zhang give the following.

Definition 2.1 ([24, Definition 2.2, Definition 2.3, Lemma 2.2]). An almost-complex structure $J$ on $M$ is said to be:

- $\mathcal{C}^{\infty}$-pure if

$$
H_{J}^{(2,0),(0,2)}(M)_{\mathbb{R}} \cap H_{J}^{(1,1)}(M)_{\mathbb{R}}=\{[0]\} ;
$$

- $\mathcal{C}^{\infty}$-full if

$$
H_{J}^{(2,0),(0,2)}(M)_{\mathbb{R}}+H_{J}^{(1,1)}(M)_{\mathbb{R}}=H_{d R}^{2}(M ; \mathbb{R}) ;
$$

- $\mathcal{C}^{\infty}$-pure-and-full if it is both $\mathcal{C}^{\infty}$-pure and $\mathcal{C}^{\infty}$-full, i.e. if the following decomposition holds:

$$
H_{J}^{(2,0),(0,2)}(M)_{\mathbb{R}} \oplus H_{J}^{(1,1)}(M)_{\mathbb{R}}=H_{d R}^{2}(M ; \mathbb{R}) .
$$

For a complex manifold $M$, by saying that $M$ is, for example, $\mathcal{C}^{\infty}$-pureand-full, we mean that the integrable almost-complex structure naturally associated with it is $\mathcal{C}^{\infty}$-pure-and-full. 
We also use the following notations:

- by saying that $J$ is complex- $\mathcal{C}^{\infty}$-pure we mean that the sum

$$
H_{J}^{(2,0)}(M)+H_{J}^{(1,1)}(M)+H_{J}^{(0,2)}(M)
$$

is direct;

- by saying that $J$ is complex- $\mathcal{C}^{\infty}$-full we mean that the equality

$$
H_{d R}^{2}(M ; \mathbb{C})=H_{J}^{(2,0)}(M)+H_{J}^{(1,1)}(M)+H_{J}^{(0,2)}(M)
$$

holds;

- by saying that $J$ is complex-C $\mathcal{C}^{\infty}$-pure-and-full we mean that $J$ induces the decomposition

$$
H_{d R}^{2}(M ; \mathbb{C})=H_{J}^{(2,0)}(M) \oplus H_{J}^{(1,1)}(M) \oplus H_{J}^{(0,2)}(M) .
$$

Remark 2.1. While being complex- $\mathcal{C}^{\infty}$-full is a stronger condition that being $\mathcal{C}^{\infty}$-full, one has to assume $J$ to be integrable to have that complex$\mathcal{C}^{\infty}$-pure condition implies the $\mathcal{C}^{\infty}$-pure one. Note also that if $J$ is $\mathcal{C}^{\infty}$-pure then

$$
H_{J}^{(1,1)}(M) \cap\left(H_{J}^{(2,0)}(M)+H_{J}^{(0,2)}(M)\right)=\{[0]\} .
$$

Moreover, we say that $J$ is $\mathcal{C}^{\infty}$-pure-and-full at the $k$-th stage if $J$ induces a decomposition of $H_{d R}^{k}(M ; \mathbb{R})$; for $k=2$, we recover the previous definitions.

Using the complex of currents instead of the complex of forms and the de Rham homology instead of the de Rham cohomology, one can define analogous concepts dually. Recall that the space of currents of dimension $k$ (or degree $2 n-k$ ) is the topological dual of $\wedge^{k} M$ : we denote it with $\mathcal{D}_{k} M:=: \mathcal{D}^{2 n-k} M$; we refer to $[\mathbf{9}, \mathbf{1 1}]$ as general references for the study of currents. Dually, the exterior differential d on $\wedge^{\bullet} M$ induces a differential on $\mathcal{D} \cdot M$, that we denote again as d; we call de Rham homology $H_{\bullet}(M ; \mathbb{R})$ the cohomology of the differential complex $(\mathcal{D} \bullet M, \mathrm{~d})$; we remember that $H_{d R}^{k}(M ; \mathbb{R}) \simeq H_{2 n-k}(M ; \mathbb{R})$. As $J$ induces a bigraduation on $\wedge^{\bullet}(M ; \mathbb{C})$, so $\mathcal{D}_{p, q} M$ are defined.

Therefore, let $H_{(2,0),(0,2)}^{J}(M)_{\mathbb{R}}$ (respectively, $\left.H_{(1,1)}^{J}(M)_{\mathbb{R}}\right)$ be the subspace of $H_{2}(M ; \mathbb{R})$ given by the homology classes represented by a current of bidimension $(2,0)+(0,2)$ (respectively, $(1,1))$. We recall the following definition by Li and Zhang (see $[\mathbf{2 4}]$ ).

Definition 2.2 ([24, Definition 2.5, Lemma 2.7]). An almost-complex structure $J$ on $M$ is said to be:

- $p u r e$ if

$$
H_{(2,0),(0,2)}^{J}(M)_{\mathbb{R}} \cap H_{(1,1)}^{J}(M)_{\mathbb{R}}=\{[0]\}
$$

- full if

$$
H_{(2,0),(0,2)}^{J}(M)_{\mathbb{R}}+H_{(1,1)}^{J}(M)_{\mathbb{R}}=H_{2}(M ; \mathbb{R})
$$


- pure-and-full if it is both pure and full, i.e. if the following decomposition holds:

$$
H_{(2,0),(0,2)}^{J}(M)_{\mathbb{R}} \oplus H_{(1,1)}^{J}(M)_{\mathbb{R}}=H_{2}(M ; \mathbb{R}) .
$$

The relations between being $\mathcal{C}^{\infty}$-pure-and-full and being pure-and-full are summarized in the following.

Theorem 2.1 (see also [24, Proposition 2.5]). The following relations between $\mathcal{C}^{\infty}$-pure-and-full and pure-and-full concepts hold:

$\mathcal{C}^{\infty}$-full at the $k$-th stage $\Longrightarrow$ pure at the $k$-th stage

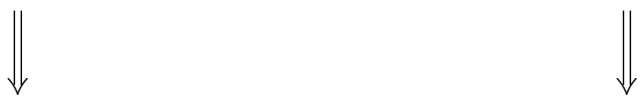

full at the $(2 n-k)$-th stage $\Longrightarrow \mathcal{C}^{\infty}$-pure at the $(2 n-k)$-th stage

Proof. First, we prove that if $J$ is $\mathcal{C}^{\infty}$-full at the $k$-th stage then it is also pure at the $k$-th stage; for the sake of simplicity, we assume $k=2$. Let

$$
\langle\cdot, \cdot \cdot\rangle: H_{d R}^{2}(M ; \mathbb{R}) \rightarrow H_{2}(M ; \mathbb{R})
$$

the non-degenerate duality paring. Let $\mathfrak{c} \in H_{(2,0),(0,2)}^{J}(M)_{\mathbb{R}} \cap H_{(1,1)}^{J}(M)_{\mathbb{R}}$, with $\mathfrak{c} \neq[0]$. Obviously, $\langle\mathfrak{c}, \cdot\rangle \bigsqcup_{H_{J}^{(2,0),(0,2)}(M)_{\mathbb{R}}}=0$ and $\langle\mathfrak{c}, \cdot\rangle \bigsqcup_{H_{J}^{(1,1)}(M)_{\mathbb{R}}}=0$; since $J$ is $\mathcal{C}^{\infty}$-full, it follows that $\mathfrak{c}=[0]$. The same argument works to prove that a full $J$ is also $\mathcal{C}^{\infty}$-pure.

To conclude the proof, we have to prove the two vertical arrows, namely that

$$
\mathcal{C}^{\infty} \text {-full at the } k \text { th stage } \stackrel{?}{\Rightarrow} \text { full at the }(2 n-k) \text {-th stage }
$$

and that

$$
\text { pure at the } k \text { th stage } \stackrel{?}{\Rightarrow} \mathcal{C}^{\infty} \text {-pure at the }(2 n-k) \text {-th stage. }
$$

Recall that a form of degree $k$ can be viewed as a current of dimension $2 n-k$ (and degree $k$ ), by means of the map

$$
T: \wedge^{k} M \rightarrow \mathcal{D}_{2 n-k} M, \quad \varphi \mapsto T_{\varphi}(\cdot) \stackrel{\text { def }}{=} \int_{M} \varphi \wedge \cdot
$$

Holding $T_{\mathrm{d}} .=\mathrm{d} T$., this map induces the inclusion

$$
H_{J}^{(p, q)}(M)_{\mathbb{R}} \hookrightarrow H_{(n-p, n-q)}^{J}(M)_{\mathbb{R}} ;
$$

being $H_{d R}^{k}(M ; \mathbb{R}) \simeq H_{2 n-k}(M ; \mathbb{R})$, the statements follow.

To prove that $\mathcal{C}^{\infty}$-full $\Rightarrow$ pure, see also [20, Theorem 3.7].

A link between $H_{d R}^{2}(M ; \mathbb{R})$ and $H_{d R}^{2 n-2}(M ; \mathbb{R})$ could provide further relations between $\mathcal{C}^{\infty}$-pure-and-full and pure-and-full notions. This is the matter of the following results, proved in $[\mathbf{2 0}]$. 
Theorem 2.2 ([20, Theorem 3.7]). Let $g$ be a Hermitian metric on $(M, J)$. If the harmonic representatives of the classes in $H_{d R}^{2}(M ; \mathbb{R})$ are of pure degree, then $J$ is both $\mathcal{C}^{\infty}$-pure-and-full and pure-and-full.

On a symplectic $2 n$-manifold $(M, \omega)$, a link between $H_{d R}^{2}(M ; \mathbb{R})$ and $H_{d R}^{2 n-2}(M ; \mathbb{R})$ could be provided if the Hard Lefschetz Condition holds; recall that $(M, \omega)$ is said to satisfy the Hard Lefschetz Condition (HLC) if, for every $k \in\{0, \ldots, n\}$, the isomorphism

$$
\left[\omega^{k}\right]: H_{d R}^{n-k}(M ; \mathbb{R}) \stackrel{\simeq}{\longrightarrow} H_{d R}^{n+k}(M ; \mathbb{R}) .
$$

holds.

Theorem 2.3 ([20, Theorem 4.1]). Let $\omega$ be a symplectic form on $M$ satisfying (HLC) and let $J$ an $\omega$-compatible almost-complex structure on $M$. If $J$ is $\mathcal{C}^{\infty}$-pure-and-full, then it is also pure-and-full.

We give now a class of examples of $\mathcal{C}^{\infty}$-pure-and-full and pure-and-full manifolds. Clearly, compact Kähler manifolds are $\mathcal{C}^{\infty}$-pure-and-full at every stage (and then also pure-and-full at every stage). Furthermore, the following theorem holds.

Theorem 2.4 (see $[\mathbf{1 3}, \mathbf{2 4}]$ ). Let $M$ be a compact complex manifold; if the Hodge-Frölicher spectral sequence degenerates at the first step and there is a weight 2 formal Hodge decomposition, then $M$ is $\mathcal{C}^{\infty}$-pure-and-full.

For the proof, see [13, Theorem 2.16, Proposition 2.17].

As a consequence of the last theorem, we have that

(1) the compact complex surfaces,

(2) the compact complex manifolds satisfying the $\partial \bar{\partial}$-Lemma (i.e., the compact complex manifolds for which every $\partial$-closed, $\bar{\partial}$-closed and d-exact form is also $\partial \bar{\partial}$-exact)

(3) and the compact complex manifolds admitting a Kähler structure

are $\mathcal{C}^{\infty}$-pure-and-full manifolds.

Indeed, for (1) we have that the assumptions of Theorem 2.4 hold by [4, Theorem 2.6], while for (2) they are satisfied by [10, Section 5.21]; finally, for (3) we have that a compact complex manifold admitting a Kähler metric satisfies the $\partial \bar{\partial}$-Lemma, see [10, Sec 5.11].

Actually, Draghici et al. proved the following.

Theorem 2.5 ([13, Theorem 2.3]). Every almost-complex structure on a compact four-manifold is $\mathcal{C}^{\infty}$-pure-and-full as well as pure-and-full.

This turns our attention to the six-dimensional case. 
Example 2.1. Let $G$ be the Lie group of matrices of the following form

$$
A=\left(\begin{array}{llllll}
e^{x_{1}} & 0 & x_{2} e^{x_{1}} & 0 & 0 & x_{3} \\
0 & e^{-x_{1}} & 0 & x_{2} e^{-x_{1}} & 0 & x_{4} \\
0 & 0 & e^{x_{1}} & 0 & 0 & x_{5} \\
0 & 0 & 0 & e^{-x_{1}} & 0 & x_{6} \\
0 & 0 & 0 & 0 & 0 & x_{1} \\
0 & 0 & 0 & 0 & 0 & 1
\end{array}\right)
$$

for $x_{1}, \ldots, x_{6} \in \mathbb{R}$. Then $G$ is a six-dimensional simply connected completely solvable Lie group. According to Fernández et al. [16], there exists a uniform discrete subgroup $\Gamma \subset G$, so that $M=\Gamma \backslash G$ is a six-dimensional compact solvmanifold. The following 1-forms on $G$

$$
\begin{aligned}
& e^{1}=d x_{1}, \quad e^{2}=d x_{2}, \quad e^{3}=\exp \left(-x_{1}\right)\left(d x_{3}-x_{2} d x_{5}\right) \\
& e^{4}=\exp \left(x_{1}\right) \quad e^{5}=\exp \left(-x_{1}\right) d x_{5}, \quad e^{6}=\exp \left(x_{1}\right) d x_{6} \\
& \times\left(d x_{4}-x_{2} d x_{6}\right),
\end{aligned}
$$

give rise to 1 -forms on $M$. We immediately obtain that

$$
\begin{aligned}
& \mathrm{d} e^{1}=0, \quad \mathrm{~d} e^{2}=0, \quad \mathrm{~d} e^{3}=-e^{1} \wedge e^{3}-e^{2} \wedge e^{5}, \\
& \mathrm{~d} e^{4}=e^{1} \wedge e^{4}-e^{2} \wedge e^{6}, \quad \mathrm{~d} e^{5}=-e^{1} \wedge e^{5}, \quad \mathrm{~d} e^{6}=e^{1} \wedge e^{6} .
\end{aligned}
$$

Since $G$ is completely solvable, in view of the Hattori theorem (see [21]), we easily obtain by (2.1), that

$$
H^{2}(M ; \mathbb{R})=\operatorname{span}_{\mathbb{R}}\left\{e^{1} \wedge e^{2}, e^{5} \wedge e^{6}, e^{3} \wedge e^{6}+e^{4} \wedge e^{5}\right\} .
$$

Therefore, setting

$$
\left\{\begin{array}{l}
\varphi^{1}=e^{1}+i e^{2} \\
\varphi^{2}=e^{3}+i e^{4} \\
\varphi^{3}=e^{5}+i e^{6}
\end{array}\right.
$$

we have that the almost-complex structure $J$ whose complex forms of type $(1,0)$ are $\varphi^{1}, \varphi^{2}, \varphi^{3}$ is $\mathcal{C}^{\infty}$-full. Indeed,

$$
\begin{array}{ll}
H_{J}^{(1,1)}(M)_{\mathbb{R}} & =\operatorname{span}_{\mathbb{R}}\left\{-\frac{1}{2 i} \varphi^{1} \wedge \bar{\varphi}^{1},-\frac{1}{2 i} \varphi^{3} \wedge \bar{\varphi}^{3}\right\}, \\
H_{J}^{(2,0),(0,2)}(M)_{\mathbb{R}} & =\operatorname{span}_{\mathbb{R}}\left\{\frac{1}{2 i}\left(\varphi^{2} \wedge \varphi^{3}-\bar{\varphi}^{2} \wedge \bar{\varphi}^{3}\right)\right\} .
\end{array}
$$

According to Theorem 2.2, since the harmonic representatives are of pure type, $J$ is both $\mathcal{C}^{\infty}$-pure-and-full and pure-and-full. 


\section{Instability along curves of complex structures}

In this section, we will show that the condition to be $\mathcal{C}^{\infty}$-pure-and-full for a complex structure is not stable under small deformations. In order to do this, we will consider the Iwasawa manifold, showing that there are curves of complex structures that are not $\mathcal{C}^{\infty}$-pure-and-full.

We first recall the definition of the Iwasawa manifold and some of its properties, see e. g. $[\mathbf{1 5}, \mathbf{2 6}]$.

On $\mathbb{C}^{3}$, consider the product $*$ defined as

$$
\left(z_{1}, z_{2}, z_{3}\right) *\left(w_{1}, w_{2}, w_{3}\right) \stackrel{\text { def }}{=}\left(z_{1}+w_{1}, z_{2}+w_{2}, z_{3}+z_{1} w_{2}+w_{3}\right) .
$$

It is immediate to check that $\left(\mathbb{C}^{3}, *\right)$ is a nilpotent Lie group isomorphic to

$$
\mathbb{H}(3) \stackrel{\text { def }}{=}\left\{\left(\begin{array}{ccc}
1 & z_{1} & z_{3} \\
0 & 1 & z_{2} \\
0 & 0 & 1
\end{array}\right) \in \operatorname{GL}(3 ; \mathbb{C}) \mid z_{1}, z_{2}, z_{3} \in \mathbb{C}\right\} .
$$

We have that $(\mathbb{Z}[\mathrm{i}])^{3} \subset \mathbb{C}^{3}$ is a cocompact discrete subgroup of $\left(\mathbb{C}^{3}, *\right)$. The Iwasawa manifold $X$ is defined as the manifold

$$
X \stackrel{\text { def }}{=}(\mathbb{Z}[\mathrm{i}])^{3} \backslash\left(\mathbb{C}^{3}, *\right) .
$$

$X$ is a compact complex three-dimensional nilmanifold; by Fernández and Gray [15], it follows that $X$ is not formal; hence, it has no Kähler metrics, see [10, Main Theorem]; nevertheless, there exists a balanced metric on $X$.

We will need the following results on the cohomology of solvmanifolds. The Hattori-Nomizu theorem states that if $M=\Gamma \backslash G$ is a compact nilmanifold (or, more in general, a compact completely solvable solvmanifold, i.e. a compact solvmanifold such that, for every $\xi$ in the Lie algebra $\mathfrak{g}$ of $G$, all the eigenvalues of $\operatorname{ad}_{\xi}$ are real) then

$$
H_{d R}^{\bullet}(M ; \mathbb{R}) \simeq H^{\bullet}\left(\wedge^{\bullet} \mathfrak{g}^{*}, \mathrm{~d}\right)
$$

(see $[\mathbf{2 1}, \mathbf{2 7}]$ ), where the Chevalley-Eilenberg cohomology $H^{\bullet}\left(\wedge^{\bullet} \mathfrak{g}^{*}\right.$, d) is the cohomology of the complex $\wedge^{\bullet} \mathfrak{g}^{*}$ endowed with the differential inherited from $\wedge^{\bullet} M$; equivalently, $H^{\bullet}\left(\wedge^{\bullet} \mathfrak{g}^{*}, \mathrm{~d}\right)$ is the cohomology of the complex of the left-invariant forms. A similar result holds for the Dolbeault cohomology of nilmanifolds. More precisely, for a compact complex nilmanifold $M$ that is holomorphically parallelizable (i.e., with trivial holomorphic tangent bundle) or whose integrable almost-complex structure $J$ is rational (i.e., such that $J\left[\mathfrak{g}_{\mathbb{Q}}\right] \subseteq \mathfrak{g}_{\mathbb{Q}}$, where $\mathfrak{g}_{\mathbb{Q}}$ is a rational Lie subalgebra of $\mathfrak{g}$ such that $\left.\mathfrak{g}=\mathfrak{g}_{\mathbb{Q}} \otimes \mathbb{R}\right)$ or whose $J$ is obtained as a small deformation of a rational one, the following isomorphism holds:

$$
H_{\bar{\partial}}^{p, q}(M) \simeq H^{q}\left(\wedge^{p, \bullet}\left(\mathfrak{g}^{\mathbb{C}}\right)^{*}, \bar{\partial}\right)
$$


(see $[\mathbf{8}, \mathbf{2 8}])$. In particular, (3.1) and (3.2) hold for the Iwasawa manifold and for its small deformations.

Let $\left(z^{i}\right)_{i \in\{1,2,3\}}$ be the standard complex coordinate system on $\mathbb{C}^{3}$; the following (1,0)-forms on $\mathbb{C}^{3}$ are invariant for the action (on the left) of $(\mathbb{Z}[\mathrm{i}])^{3}$, so they give rise to a global coframe for $T^{* 1,0} X$ :

$$
\left\{\begin{array}{l}
\varphi^{1} \stackrel{\text { def }}{=} \mathrm{d} z^{1} \\
\varphi^{2} \stackrel{\text { def }}{=} \mathrm{d} z^{2} \\
\varphi^{3} \stackrel{\text { def }}{=} \mathrm{d} z^{3}-z^{1} \mathrm{~d} z^{2}
\end{array} .\right.
$$

The structure equations are therefore

$$
\left\{\begin{array}{l}
\mathrm{d} \varphi^{1}=0 \\
\mathrm{~d} \varphi^{2}=0 \\
\mathrm{~d} \varphi^{3}=-\varphi^{1} \wedge \varphi^{2}
\end{array} .\right.
$$

By Hattori-Nomizu theorem, we compute the real cohomology algebra of $X$ (for simplicity, we list the harmonic representative instead of its class and write $\varphi^{A \bar{B}}$ for $\varphi^{A} \wedge \bar{\varphi}^{B}$ and so on):

$$
\begin{aligned}
H_{d R}^{1}(X ; \mathbb{R})= & \operatorname{span}_{\mathbb{R}}\left\{\varphi^{1}+\bar{\varphi}^{1}, \mathrm{i}\left(\varphi^{1}-\bar{\varphi}^{1}\right), \varphi^{2}+\bar{\varphi}^{2}, \mathrm{i}\left(\varphi^{2}-\bar{\varphi}^{2}\right)\right\}, \\
H_{d R}^{2}(X ; \mathbb{R})= & \operatorname{span}_{\mathbb{R}}\left\{\varphi^{13}+\varphi^{\overline{1} \overline{3}}, \mathrm{i}\left(\varphi^{13}-\varphi^{\overline{1} \overline{3}}\right), \varphi^{23}+\varphi^{\overline{2} \overline{3}},\right. \\
& \left.\mathrm{i}\left(\varphi^{23}-\varphi^{\overline{2} \overline{3}}\right), \varphi^{1 \overline{2}}-\varphi^{2 \overline{1}}, \mathrm{i}\left(\varphi^{1 \overline{2}}+\varphi^{2 \overline{1}}\right), \mathrm{i} \varphi^{1 \overline{1}}, \mathrm{i} \varphi^{2 \overline{2}}\right\}, \\
H_{d R}^{3}(X ; \mathbb{R})= & \operatorname{span}_{\mathbb{R}}\left\{\varphi^{123}+\varphi^{\overline{1} \overline{2} \overline{3}}, \mathrm{i}\left(\varphi^{123}-\varphi^{\overline{1} \overline{2} \overline{3}}\right), \varphi^{13 \overline{1}}+\varphi^{1 \overline{1} \overline{3}},\right. \\
& \mathrm{i}\left(\varphi^{13 \overline{1}}-\varphi^{1 \overline{1} \overline{3}}\right), \varphi^{13 \overline{2}}+\varphi^{2 \overline{1} \overline{3}}, \mathrm{i}\left(\varphi^{13 \overline{2}}-\varphi^{2 \overline{1} \overline{3}}\right), \varphi^{23 \overline{1}}+\varphi^{1 \overline{2} \overline{3}}, \\
& \left.\mathrm{i}\left(\varphi^{23 \overline{1}}-\varphi^{1 \overline{2} \overline{3}}\right), \varphi^{23 \overline{2}}+\varphi^{2 \overline{2} \overline{3}}, \mathrm{i}\left(\varphi^{23 \overline{2}}-\varphi^{2 \overline{2} \overline{3}}\right)\right\} .
\end{aligned}
$$

Note that each harmonic representative is of pure degree. The Betti numbers of $X$ are

$$
b^{0}=1, \quad b^{1}=4, \quad b^{2}=8, \quad b^{3}=10 .
$$

Nakamura in $[\mathbf{2 6}]$ computed the small deformations $\left\{X_{\mathbf{t}}\right\}_{\mathbf{t} \in \Delta(\mathbf{0}, \varepsilon) \subseteq \mathbb{C}^{6}}$ of the Iwasawa manifold $X$ : by [26, page 95], a local system of complex coordinates for the complex structure at $\mathbf{t}=\left(t_{11}, t_{12}, t_{21}, t_{22}, t_{31}, t_{32}\right) \in \mathbb{C}^{6}$ is 
given by

$$
\left\{\begin{array}{l}
\zeta_{\mathbf{t}}^{1}=z^{1}+\sum_{j=1}^{2} t_{1 j} \bar{z}^{j} \\
\zeta_{\mathbf{t}}^{2}=z^{2}+\sum_{j=1}^{2} t_{2 j} \bar{z}^{j} \\
\zeta_{\mathbf{t}}^{3}=z^{3}+\sum_{j=1}^{2}\left(t_{3 j}+t_{2 j} z^{1}\right) \bar{z}^{j}+A(\overline{\mathbf{z}})-D(\mathbf{t}) \bar{z}^{3}
\end{array},\right.
$$

where

$$
\begin{aligned}
& A(\overline{\mathbf{z}}) \stackrel{\text { def }}{=} \frac{1}{2}\left(t_{11} t_{21} \bar{z}^{1} \bar{z}^{1}+2 t_{11} t_{22} \bar{z}^{1} \bar{z}^{2}+t_{12} t_{22} \bar{z}^{2} \bar{z}^{2}\right), \\
& D(\mathbf{t}) \stackrel{\text { def }}{=} t_{11} t_{22}-t_{12} t_{21} .
\end{aligned}
$$

Nakamura also computed the numerical characters of these deformations, dividing them into three classes according to their Hodge diamond:

\begin{tabular}{c||cc|ccc|cccc} 
& $h^{1,0}$ & $h^{0,1}$ & $h^{2,0}$ & $h^{1,1}$ & $h^{0,2}$ & $h^{3,0}$ & $h^{2,1}$ & $h^{1,2}$ & $h^{0,3}$ \\
\hline (i) & 3 & 2 & 3 & 6 & 2 & 1 & 6 & 6 & 1 \\
(ii) & 2 & 2 & 2 & 5 & 2 & 1 & 5 & 5 & 1 \\
(iii) & 2 & 2 & 1 & 5 & 2 & 1 & 4 & 4 & 1
\end{tabular}

More exactly, the classes are characterized by the following values of the parameters:

class (i): $t_{11}=t_{12}=t_{21}=t_{22}=0 ;$

class (ii): $D(\mathbf{t})=0$ but $\left(t_{11}, t_{12}, t_{21}, t_{22}\right) \neq(0,0,0,0)$;

class (iii): $D(\mathbf{t}) \neq 0$.

Note that the Hodge diamond of the deformations of the class (i) is the same of the Iwasawa manifold, while deformations of the class (iii) have the Hodge-Frölicher spectral sequence that degenerates at the first step. Note also that the table above proves that the Hodge numbers are not stable under small deformations, [26, Theorem 2], in contrast with the Kähler case.

Equivalently, $X_{\mathbf{t}}$ could be viewed as $\mathbb{C}^{3} / \Gamma_{\mathbf{t}}$ where $\Gamma_{\mathbf{t}}$ is the group generated by the transformations

$$
\left\{\begin{aligned}
\zeta^{\prime 1}:=\zeta^{1}+\left(\omega_{1}+\sum_{j=1}^{2} t_{1 j} \bar{\omega}_{j}\right) \\
\zeta^{\prime 2}:=\zeta^{2}+\left(\omega_{2}+\sum_{j=1}^{2} t_{2 j} \bar{\omega}_{j}\right) \\
\zeta^{\prime 3}:=\zeta^{3}+\left(\omega_{3}+\sum_{j=1}^{2} t_{3 j} \bar{\omega}_{j}\right)+\omega_{1} \zeta^{2}+\left(\sum_{j=1}^{2} t_{2 j} \bar{\omega}_{j}\right)\left(\zeta^{1}+\omega_{1}\right) \\
\quad+A(\bar{\omega})-D(\mathbf{t}) \bar{\omega}_{3}
\end{aligned}\right.
$$

varying $\omega:=:\left(\omega_{1}, \omega_{2}, \omega_{3}\right) \in(\mathbb{Z}[\mathrm{i}])^{3}$. 
In the sequel, $J$ will denote the integrable almost-complex structure associated to $X$ and $J_{\mathbf{t}}$ will denote the one associated to $X_{\mathbf{t}}$.

Now, we can prove the following.

Theorem 3.1. Let $X:=(\mathbb{Z}[\mathrm{i}])^{3} \backslash\left(\mathbb{C}^{3}, *\right)$ be the Iwasawa manifold. Then:

- $X$ is $\mathcal{C}^{\infty}$-pure-and-full at every stage as well as pure-and-full at every stage;

- the small deformations of $X$ of the class (i) are $\mathcal{C}^{\infty}$-pure-and-full and pure-and-full at every stage;

- the small deformations of $X$ of the classes (ii) and (iii) are neither $\mathcal{C}^{\infty}$-pure nor $\mathcal{C}^{\infty}$-full nor pure nor full.

Proof. We divide the proof in various steps.

Step 1. $X$ is a $\mathcal{C}^{\infty}$-pure-and-full manifold at every stage.

Since harmonic representatives in $H_{d R}^{\bullet}(X ; \mathbb{R})$ are of pure degree, the statement follows from Theorem 2.2.

Step 2. Small deformations of the class (i) remain $\mathcal{C}^{\infty}$-pure-and-full at every stage.

A coframe of $(1,0)$-forms invariant for the action of $\Gamma_{\mathbf{t}}$ on $\mathbb{C}^{3}$ is given by

$$
\left\{\begin{array}{l}
\varphi_{\mathbf{t}}^{1} \stackrel{\text { def }}{=} \mathrm{d} \zeta_{\mathbf{t}}^{1} \\
\varphi_{\mathbf{t}}^{2} \stackrel{\text { def }}{=} \mathrm{d} \zeta_{\mathbf{t}}^{2} \\
\varphi_{\mathbf{t}}^{3} \stackrel{\text { def }}{=} \mathrm{d} \zeta_{\mathbf{t}}^{3}-\zeta_{\mathbf{t}}^{1} \mathrm{~d} \zeta_{\mathbf{t}}^{2}
\end{array} .\right.
$$

Hence, $\left\{\varphi_{\mathbf{t}}^{1}, \varphi_{\mathbf{t}}^{2}, \varphi_{\mathbf{t}}^{3}\right\}$ satisfies the same structure equations as $\left\{\varphi^{1}, \varphi^{2}, \varphi^{3}\right\}$. Therefore, the same argument in Step 1 applies to deformations of such a class.

Step 3. Computation of the structure equations for small deformations of the class (ii).

Consider the system of complex coordinates given by

$$
\left\{\begin{array}{l}
\zeta_{\mathbf{t}}^{1} \stackrel{\text { def }}{=} z^{1}+\sum_{\lambda=1}^{2} t_{1 \lambda} \bar{z}^{\lambda} \\
\zeta_{\mathbf{t}}^{2} \stackrel{\text { def }}{=} z^{2}+\sum_{\lambda=1}^{2} t_{2 \lambda} \bar{z}^{\lambda} \\
\zeta_{\mathbf{t}}^{3} \stackrel{\text { def }}{=} z^{3}+\sum_{\lambda=1}^{2}\left(t_{3 \lambda}+t_{2 \lambda} z^{1}\right) \bar{z}^{\lambda}+A(\bar{z})
\end{array} .\right.
$$

A straightforward computation gives

$$
\left\{\begin{array}{l}
z^{1}=\gamma\left(\zeta_{\mathbf{t}}^{1}+\lambda_{1} \bar{\zeta}_{\mathbf{t}}^{1}+\lambda_{2} \zeta_{\mathbf{t}}^{2}+\lambda_{3} \bar{\zeta}_{\mathbf{t}}^{2}\right) \\
z^{2}=\alpha\left(\mu_{0} \zeta_{\mathbf{t}}^{1}+\mu_{1} \bar{\zeta}_{\mathbf{t}}^{1}+\mu_{2} \zeta_{\mathbf{t}}^{2}+\mu_{3} \bar{\zeta}_{\mathbf{t}}^{2}\right)
\end{array}\right.
$$

where $\alpha, \beta, \gamma, \lambda_{i}$ (for $i \in\{1,2,3\}$ ), $\mu_{j}$ (for $j \in\{0,1,2,3\}$ ) are complex constants depending only on $\mathbf{t}=\left(t_{11}, t_{12}, t_{21}, t_{22}, t_{31}, t_{32}\right) \in \mathbb{C}^{6}$ and 
defined as follows:

$$
\left\{\begin{array}{l}
\alpha \stackrel{\text { def }}{=} \frac{1}{1-\left|t_{22}\right|^{2}-t_{21} \bar{t}_{12}} \\
\beta \stackrel{\text { def }}{=} t_{21} \bar{t}_{11}+t_{22} \bar{t}_{21} \\
\gamma \stackrel{\text { def }}{=} \frac{1}{1-\left|t_{11}\right|^{2}-\alpha \beta\left(t_{11} \bar{t}_{12}+t_{12} \bar{t}_{22}\right)-t_{12} \bar{t}_{21}} \\
\lambda_{1} \stackrel{\text { def }}{=}-t_{11}\left(1+\alpha \bar{t}_{12} t_{21}+\alpha\left|t_{22}\right|^{2}\right) \\
\lambda_{2} \stackrel{\text { def }}{=} \alpha\left(t_{11} \bar{t}_{12}+t_{12} \bar{t}_{22}\right) \\
\lambda_{3} \stackrel{\text { def }}{=}-t_{12}\left(1+\alpha \bar{t}_{12} t_{21}+\alpha\left|t_{22}\right|^{2}\right) \\
\mu_{0} \stackrel{\text { def }}{=} \beta \gamma \\
\mu_{1} \stackrel{\text { def }}{=} \lambda_{1} \beta \gamma-t_{21} \\
\mu_{2} \stackrel{\text { def }}{=} 1+\lambda_{2} \beta \gamma \\
\mu_{3} \stackrel{\text { def }}{=} \lambda_{3} \beta \gamma-t_{22}
\end{array} .\right.
$$

Consider the (1,0)-forms invariant for the action of $\Gamma_{\mathbf{t}}$ on $\mathbb{C}^{3}$ given by

$$
\left\{\begin{array}{l}
\varphi_{\mathbf{t}}^{1} \stackrel{\text { def }}{=} \mathrm{d} \zeta_{\mathbf{t}}^{1} \\
\varphi_{\mathbf{t}}^{2} \stackrel{\text { def }}{=} \mathrm{d} \zeta_{\mathbf{t}}^{2} \\
\varphi_{\mathbf{t}}^{3} \stackrel{\text { def }}{=} \mathrm{d} \zeta_{\mathbf{t}}^{3}-z^{1} \mathrm{~d} \zeta_{\mathbf{t}}^{2}-\left(t_{21} \bar{z}^{1}+t_{22} \bar{z}^{2}\right) \mathrm{d} \zeta_{\mathbf{t}}^{1}
\end{array} ;\right.
$$

we could now easily compute the structure equations:

$$
\left\{\begin{aligned}
\mathrm{d} \varphi_{\mathbf{t}}^{1}= & 0 \\
\mathrm{~d} \varphi_{\mathbf{t}}^{2}= & 0 \\
\mathrm{~d} \varphi_{\mathbf{t}}^{3}= & \sigma_{12} \varphi_{\mathbf{t}}^{1} \wedge \varphi_{\mathbf{t}}^{2}+\sigma_{1 \overline{1}} \varphi_{\mathbf{t}}^{1} \wedge \bar{\varphi}_{\mathbf{t}}^{1}+\sigma_{1 \overline{2}} \varphi_{\mathbf{t}}^{1} \wedge \bar{\varphi}_{\mathbf{t}}^{2} \\
& +\sigma_{2 \overline{1}} \varphi_{\mathbf{t}}^{2} \wedge \bar{\varphi}_{\mathbf{t}}^{1}+\sigma_{2 \overline{2}} \varphi_{\mathbf{t}}^{2} \wedge \bar{\varphi}_{\mathbf{t}}^{2}
\end{aligned}\right.
$$

where $\sigma_{12}, \sigma_{1 \overline{1}}, \sigma_{1 \overline{2}}, \sigma_{2 \overline{1}}, \sigma_{2 \overline{2}}$ are given by

$$
\left\{\begin{array}{l}
\sigma_{12} \stackrel{\text { def }}{=}-\gamma+t_{21} \bar{\lambda}_{3} \bar{\gamma}+t_{22} \bar{\alpha} \bar{\mu}_{3} \\
\sigma_{1 \overline{1}} \stackrel{\text { def }}{=} t_{21} \overline{\gamma\left(1+t_{21} \bar{t}_{12} \alpha+\left|t_{22}\right|^{2} \alpha\right)} \\
\sigma_{1 \overline{2}} \stackrel{\text { def }}{=} t_{22} \overline{\gamma\left(1+t_{21} \bar{t}_{12} \alpha+\left|t_{22}\right|^{2} \alpha\right)} \\
\sigma_{2 \overline{1}} \stackrel{\text { def }}{=}-t_{11} \gamma\left(1+t_{21} \bar{t}_{12} \alpha+\left|t_{22}\right|^{2} \alpha\right) \\
\sigma_{2 \overline{2}} \stackrel{\text { def }}{=}-t_{12} \gamma\left(1+t_{21} \bar{t}_{12} \alpha+\left|t_{22}\right|^{2} \alpha\right)
\end{array} .\right.
$$


Note that, for small deformations of the class (ii), one has $\sigma_{12} \neq 0$ and $\left(\sigma_{1 \overline{1}}, \sigma_{1 \overline{2}}, \sigma_{2 \overline{1}}, \sigma_{2 \overline{2}}\right) \neq(0,0,0,0)$.

This ends Step 3.

Step 4. The small deformations of the class (ii) are neither $\mathcal{C}^{\infty}$-pure nor full.

Note that

$$
\left[\sigma_{12} \varphi_{\mathbf{t}}^{12}\right]=\left[\sigma_{1 \overline{1}} \varphi_{\mathbf{t}}^{1 \overline{1}}+\sigma_{1 \overline{2}} \varphi_{\mathbf{t}}^{1 \overline{2}}+\sigma_{2 \overline{1}} \varphi_{\mathbf{t}}^{2 \overline{1}}+\sigma_{2 \overline{2}} \varphi_{\mathbf{t}}^{2 \overline{2}}\right] \neq[0]
$$

in $H_{d R}^{2}(X ; \mathbb{C})$. Therefore,

$$
H_{J_{\mathbf{t}}}^{(1,1)}\left(X_{\mathbf{t}}\right) \cap\left(H_{J_{\mathbf{t}}}^{(2,0)}\left(X_{\mathbf{t}}\right)+H_{J_{\mathbf{t}}}^{(0,2)}\left(X_{\mathbf{t}}\right)\right) \neq\{[0]\},
$$

and in particular $X_{\mathbf{t}}$ is not complex- $\mathcal{C}^{\infty}$-pure. It follows from the fact observed at page 408 that $X_{\mathbf{t}}$ cannot be $\mathcal{C}^{\infty}$-pure; from Theorem 2.1 it follows that $X_{\mathbf{t}}$ cannot be even full.

Step 5. The small deformations of the class (ii) are neither pure nor $\mathcal{C}^{\infty}$-full.

For a fixed small $\mathbf{t}$, choose two positive constants $A$ and $B$ such that

$$
\left(A \sigma_{1 \overline{2}}-B \sigma_{1 \overline{1}}, A \sigma_{2 \overline{2}}-B \sigma_{2 \overline{1}}\right) \neq(0,0)
$$

computing $-\mathrm{d}\left(A \varphi_{\mathbf{t}}^{13 \overline{3}}+B \varphi_{\mathbf{t}}^{23 \overline{3}}\right)$, note that

$$
\begin{aligned}
& {\left[\left(A \sigma_{2 \overline{1}}-B \sigma_{1 \overline{1}}\right) \varphi_{\mathbf{t}}^{12 \overline{1} \overline{3}}+\left(A \sigma_{2 \overline{2}}-B \sigma_{1 \overline{2}}\right) \varphi_{\mathbf{t}}^{12 \overline{2} \overline{3}}-A \bar{\sigma}_{12} \varphi_{\mathbf{t}}^{13 \overline{1} \overline{2}}-B \bar{\sigma}_{12} \varphi_{\mathbf{t}}^{23 \overline{1} \overline{2}}\right]} \\
& \quad=\left[\left(A \bar{\sigma}_{1 \overline{2}}-B \bar{\sigma}_{1 \overline{1}}\right) \varphi_{\mathbf{t}}^{123 \overline{1}}+\left(A \bar{\sigma}_{2 \overline{2}}-B \bar{\sigma}_{2 \overline{1}}\right) \varphi_{\mathbf{t}}^{123 \overline{2}}\right] \neq[0]
\end{aligned}
$$

in $H_{d R}^{4}(X ; \mathbb{C})$. As before, it follows that $X_{\mathbf{t}}$ is not $\mathcal{C}^{\infty}$-pure at the fourth stage, and consequently it is not even pure nor $\mathcal{C}^{\infty}$-full, by Theorem 2.1.

Step 6. Small deformations of the class (iii) are neither $\mathcal{C}^{\infty}$-pure nor $\mathcal{C}^{\infty}$-full.

We omit the computations, since they are quite similar to those ones corresponding to the deformations of class (ii).

As a corollary of the last theorem, we obtain the following theorem of instability.

Theorem 3.2. Compact complex $\mathcal{C}^{\infty}$-pure-and-full (or $\mathcal{C}^{\infty}$-pure or $\mathcal{C}^{\infty}$-full or pure-and-full or pure or full) manifolds are not stable under small deformations of the complex structure. 
Remark 3.1. By the proof of Theorem 3.1, it follows that the numbers

$$
h_{J_{\mathbf{t}}}^{+}(X) \stackrel{\text { def }}{=} \operatorname{dim}_{\mathbb{R}} H_{J_{\mathbf{t}}}^{(1,1)}(X)_{\mathbb{R}}, \quad h_{J_{\mathbf{t}}}^{-}(X) \stackrel{\text { def }}{=} \operatorname{dim}_{\mathbb{R}} H_{J_{\mathbf{t}}}^{(2,0),(0,2)}(X)_{\mathbb{R}},
$$

where $J_{\mathbf{t}}$ is a small deformation of class (i), are equal to

$$
h_{J_{\mathbf{t}}}^{+}(X)=4, \quad h_{J_{\mathbf{t}}}^{-}(X)=4 \text {, }
$$

for $\mathbf{t}$ small enough.

We recall that for the complex deformations of a complex structure $J$ on a four-dimensional compact manifold $M$, one has that $h_{J}^{+}(M)$ and $h_{J}^{-}(M)$ are topological invariants (see [14]).

Now we show that none of the above deformed complex structures described in Theorem 3.1 can be tamed by any symplectic form $\omega$ on the Iwasawa manifold.

We start with the following:

Proposition 3.1. Let $(M, \omega)$ be a symplectic manifold. Assume that there exists an $\omega$-tamed complex structure $J$ on $M$. Denote by $\tilde{\omega}$ the fundamental form of the Hermitian metric

$$
\tilde{g}_{J}(X, Y)=\frac{1}{2}(\omega(X, J Y)+\omega(Y, J X)) .
$$

Then

$$
\partial \bar{\partial} \tilde{\omega}=0
$$

Proof. By definition, we have

$$
\tilde{\omega}=\frac{1}{2}(\omega+J \omega) .
$$

Therefore, by viewing $\omega$ and $\tilde{\omega}$ as real elements of $\wedge^{2}(M ; \mathbb{C})$, we can write

$$
\begin{aligned}
& \omega=\omega^{2,0}+\omega^{1,1}+\overline{\omega^{2,0},} \\
& \tilde{\omega}=\omega^{1,1},
\end{aligned}
$$

where $\overline{\omega^{1,1}}=\omega^{1,1}$. Hence, writing $\mathrm{d}=\partial+\bar{\partial}$, we have that

$$
\mathrm{d} \omega=0 \Leftrightarrow\left\{\begin{array}{l}
\partial \omega^{2,0}=0, \\
\partial \omega^{1,1}+\bar{\partial} \omega^{2,0}=0 .
\end{array}\right.
$$

Therefore,

$$
\partial \bar{\partial} \tilde{\omega}=\partial \bar{\partial} \omega^{1,1}=-\bar{\partial} \partial \omega^{1,1}=\bar{\partial}^{2} \omega^{2,0}=0 .
$$

Now, we can prove the following:

Theorem 3.3. Let $M=\Gamma \backslash G$ be a (non-toral) compact nilmanifold of dimension 6 endowed with an invariant complex structure $J$. Then there are no symplectic structures $\omega$ on $M$ such that $J$ is $\omega$-tamed. 
Proof. First of all we show that there are no invariant symplectic forms $\omega$ on $M$ such that $J$ is $\omega$-tamed. On the contrary, let $\omega$ be such an invariant symplectic structure. Then by Proposition 3.1, $M$ has an invariant Hermitian metric $g$ whose fundamental form $\omega$ satisfies $\partial \bar{\partial} \omega=0$. Then, by [19, Theorem 1.2 p. 320] we can find a basis of complex (1,0)-forms $\left\{\varphi^{1}, \varphi^{2}, \varphi^{3}\right\}$ for $J$ such that

$$
\left\{\begin{array}{l}
\mathrm{d} \varphi^{1}=0, \\
\mathrm{~d} \varphi^{2}=0 \\
\mathrm{~d} \varphi^{3}=A \bar{\varphi}^{1} \wedge \varphi^{2}+B \bar{\varphi}^{2} \wedge \varphi^{2}+C \varphi^{1} \wedge \bar{\varphi}^{1}+D \varphi^{1} \wedge \bar{\varphi}^{2}+E \varphi^{1} \wedge \varphi^{2},
\end{array}\right.
$$

where $A, B, C, D, E \in \mathbb{C}$. We immediately obtain

$$
\begin{aligned}
& \partial \varphi^{1}=0, \quad \partial \varphi^{2}=0, \quad \partial \varphi^{3}=E \varphi^{1} \wedge \varphi^{2}, \\
& \bar{\partial} \varphi^{1}=0, \quad \bar{\partial} \varphi^{2}=0, \quad \bar{\partial} \varphi^{3}=A \bar{\varphi}^{1} \wedge \varphi^{2}+B \bar{\varphi}^{2} \wedge \varphi^{2}+C \varphi^{1} \wedge \bar{\varphi}^{1} \\
& +D \varphi^{1} \wedge \bar{\varphi}^{2} \text {. }
\end{aligned}
$$

Write

$$
\omega=\omega^{2,0}+\omega^{1,1}+\overline{\omega^{2,0}}
$$

where

$$
\omega^{2,0}=\sum_{i<j} a_{i j} \varphi^{i} \wedge \varphi^{j}, \quad \omega^{1,1}=\frac{i}{2} \sum_{i, j=1}^{3} b_{i \bar{j}} \varphi^{i} \wedge \bar{\varphi}^{j}, \quad \omega^{1,1}=\overline{\omega^{1,1}},
$$

with $a_{i j}, b_{i \bar{j}} \in \mathbb{C}$. Then a straightforward computation by using (3.3) implies that $\mathrm{d} \omega=0$ if and only if

$$
A=B=C=D=E=0
$$

or

$$
b_{3 \overline{3}}=0 .
$$

In both cases we obtain a contradiction.

Assume that there exists a symplectic form $\omega$ on $M$ such that $J$ is $\omega$-tamed. Then one can consider

$$
\hat{\omega}(X, Y)=\int_{M} \omega_{\left.\right|_{p}}\left(X_{p}, Y_{p}\right) \eta
$$

$\eta$ being the volume form given in [25, Lemma 6.2]. Then, it can be showed (see e.g., $[\mathbf{5}, \mathbf{1 8}]$ ) that $\mathrm{d} \hat{\omega}=0$. Moreover, since $J$ is invariant, we have

$$
\begin{aligned}
\hat{\omega}(X, J X) & =\int_{M} \omega_{\left.\right|_{p}}\left(X_{p},(J X)_{p}\right) \eta \\
& =\int_{M} \omega_{\left.\right|_{p}}\left(X_{p}, J_{p} X_{p}\right) \eta>0 .
\end{aligned}
$$

Therefore (3.4) defines an invariant symplectic form $\hat{\omega}$ such that $J$ is $\hat{\omega}$-tamed. This is absurd. 
Relating to the speculation in $[\mathbf{2 4}$, p. 678] by Li and Zhang and to $[\mathbf{2 9}$, Question 1.7] raised by Streets and Tian, as a consequence of the last Theorem, we get the following:

Theorem 3.4. Let $X:=(\mathbb{Z}[\mathrm{i}])^{3} \backslash\left(\mathbb{C}^{3}, *\right)$ be the Iwasawa manifold. Then any small complex deformation of $X$ cannot be tamed by any symplectic form.

\section{Stability along curves of almost-complex structures}

The $\mathcal{C}^{\infty}$-pure-and-full property makes sense for an arbitrary almost-complex structure, even not integrable. In this section, we will study the stability of this property along curves of almost-complex structures.

Let $J$ be an almost-complex structure on $M^{2 n}$. Recall the following local result, see $[\mathbf{3}]$ : a curve $\left\{J_{t}\right\}_{t \in I \subseteq \mathbb{R}}$ of almost-complex structures through $J$ could be written, for $t \in(-\varepsilon, \varepsilon)$ with $\varepsilon>0$ sufficiently small, as

$$
J_{t}=\left(\mathrm{id}-L_{t}\right) J\left(\mathrm{id}-L_{t}\right)^{-1},
$$

where $L_{t}$ is an endomorphism of the tangent bundle such that

$$
L_{t} J+J L_{t}=0 \text {; }
$$

we could write $L_{t}=: t L+\mathrm{o}(t)$; recall also that: if $J$ is compatible with a symplectic form $\omega$, then the curves made up of $\omega$-compatible almost-complex structures $J_{t}$ are exactly those ones for which $L^{\mathrm{t}}=L$. For several examples of families constructed in such a way, see [20].

We begin with studying curves through the standard Kähler structure on the complex 2-torus, $\left(\mathbb{T}_{\mathbb{C}}^{2}, J_{0}, \omega_{0}\right)$. Let

$$
L=\left(\begin{array}{ll|ll}
\ell & & & \\
& 0 & & \\
\hline & & -\ell & \\
& & & 0
\end{array}\right),
$$

where $\ell \in \mathcal{C}^{\infty}\left(\mathbb{R}^{4} ; \mathbb{R}\right)$ is a $\mathbb{Z}^{4}$-periodic function. Defining (for small $t$ )

$$
J_{t, \ell} \stackrel{\text { def }}{=}(\mathrm{id}-t L) J(\mathrm{id}-t L)^{-1},
$$

we get the $\omega_{0}$-compatible almost-complex structure

$$
J_{t, \ell}=\left(\begin{array}{cc|cc} 
& & -\frac{1-t \ell}{1+t \ell} & \\
& & & -1 \\
\hline \frac{1+t \ell}{1-t \ell} & & &
\end{array}\right) .
$$

Set

$$
\alpha:=: \alpha(t, \ell) \stackrel{\text { def }}{=} \frac{1-t \ell}{1+t \ell} .
$$


A coframe for the holomorphic cotangent bundle is given by

$$
\left\{\begin{array}{l}
\varphi_{t}^{1}:=\mathrm{d} x^{1}+\mathrm{i} \alpha \mathrm{d} x^{3} \\
\varphi_{t}^{2}:=\mathrm{d} x^{2}+\mathrm{id} x^{4}
\end{array},\right.
$$

from which we compute

$$
\left\{\begin{array}{l}
\mathrm{d} \varphi_{t}^{1}=\mathrm{i} \mathrm{d} \alpha \wedge \mathrm{d} x^{3} \\
\mathrm{~d} \varphi_{t}^{2}=0
\end{array} ;\right.
$$

note that taking $\ell=\ell\left(x^{1}, x^{3}\right)$, the corresponding almost-complex structure $J_{t, \ell}$ is integrable, in fact $\left(J_{t, \ell}, \omega_{0}\right)$ is a Kähler structure on $\mathbb{T}_{\mathbb{C}}^{2}$. Remember that $J_{t, \ell}$ has to be $\mathcal{C}^{\infty}$-pure-and-full, $\mathbb{T}_{\mathbb{C}}^{2}$ being a four-dimensional manifold. For the sake of simplicity, we assume $\ell=\ell\left(x^{2}\right)$ not constant. Setting

$$
\begin{aligned}
& v_{1} \stackrel{\text { def }}{=} \mathrm{d} x^{1} \wedge \mathrm{d} x^{2}-\alpha \mathrm{d} x^{3} \wedge \mathrm{d} x^{4}, \\
& v_{2} \stackrel{\text { def }}{=} \mathrm{d} x^{1} \wedge \mathrm{d} x^{4}-\alpha \mathrm{d} x^{2} \wedge \mathrm{d} x^{3}, \\
& w_{1} \stackrel{\text { def }}{=} \alpha \mathrm{d} x^{1} \wedge \mathrm{d} x^{3}, \\
& w_{2} \stackrel{\text { def }}{=} \mathrm{d} x^{2} \wedge \mathrm{d} x^{4}, \\
& w_{3} \stackrel{\text { def }}{=} \mathrm{d} x^{1} \wedge \mathrm{d} x^{2}+\alpha \mathrm{d} x^{3} \wedge \mathrm{d} x^{4}, \\
& w_{4} \stackrel{\text { def }}{=} \mathrm{d} x^{1} \wedge \mathrm{d} x^{4}+\alpha \mathrm{d} x^{2} \wedge \mathrm{d} x^{3},
\end{aligned}
$$

the condition in order that an arbitrary $J_{t, \ell^{-}}$anti-invariant real 2-form $\psi=$ $A v_{1}+B v_{2}$ is closed is expressed by

$$
\left\{\begin{array}{l}
A_{3}-B_{1} \alpha=0 \\
A_{4}-B_{2}=0 \\
-A_{1} \alpha-B_{3}=0 \\
-B_{4} \alpha-A_{2} \alpha-A \alpha_{2}=0
\end{array}\right.
$$

(here and later on, we write, for example, $A_{3}$ instead of $\frac{\partial A}{\partial x^{3}}$ ). By solving (4.3), we obtain

$$
\psi=\frac{A}{\alpha} v_{1}+B v_{2} \quad \text { with } \quad A, B \in \mathbb{R} .
$$

Therefore, for small enough $t \neq 0$, according to [14], we have the uppersemicontinuity property

$$
\operatorname{dim}_{\mathbb{R}} H_{J_{t, \ell}}^{(2,0),(0,2)}\left(\mathbb{T}_{\mathbb{C}}^{2}\right)_{\mathbb{R}} \leq 2=\operatorname{dim}_{\mathbb{R}} H_{J}^{(2,0),(0,2)}\left(\mathbb{T}_{\mathbb{C}}^{2}\right)_{\mathbb{R}}
$$


from which we get the lower one

$$
\operatorname{dim}_{\mathbb{R}} H_{J_{t, \ell}}^{(1,1)}\left(\mathbb{T}_{\mathbb{C}}^{2}\right)_{\mathbb{R}} \geq 4=\operatorname{dim}_{\mathbb{R}} H_{J}^{(1,1)}\left(\mathbb{T}_{\mathbb{C}}^{2}\right)_{\mathbb{R}}
$$

Now, we turn our attention to the case of dimension greater than 4 . We construct curves through the standard Kähler structure on the complex 3-torus, $\left(\mathbb{T}_{\mathbb{C}}^{3}, J_{0}, \omega_{0}\right)$. Let

$$
L=\left(\begin{array}{lll|lll}
\ell & & & & & \\
& 0 & & & & \\
& & 0 & & & \\
\hline & & & -\ell & & \\
& & & & 0 & \\
& & & & & 0
\end{array}\right),
$$

where $\ell \in \mathcal{C}^{\infty}\left(\mathbb{R}^{6} ; \mathbb{R}\right)$ is a $\mathbb{Z}^{6}$-periodic function. As before, defining $J_{t, \ell}$ (for small $t$ ) as in (4.2), we get the $\omega_{0}$-compatible almost-complex structure

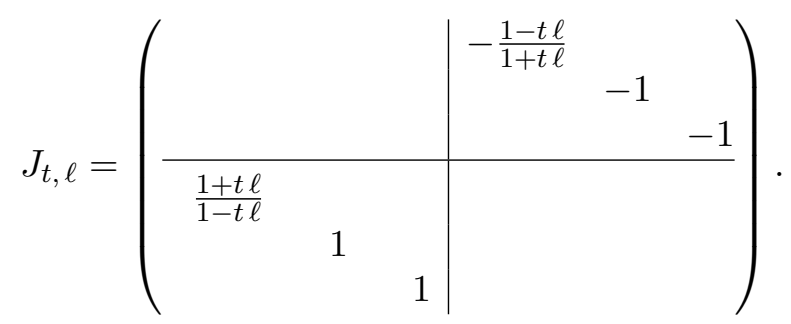

As before, setting

$$
\alpha:=: \alpha(t, \ell) \stackrel{\text { def }}{=} \frac{1-t \ell}{1+t \ell},
$$

it follows that a coframe for the holomorphic cotangent bundle is given by

$$
\left\{\begin{array}{l}
\varphi_{t}^{1}:=\mathrm{d} x^{1}+\mathrm{i} \alpha \mathrm{d} x^{4} \\
\varphi_{t}^{2}:=\mathrm{d} x^{2}+\mathrm{id} x^{5} \\
\varphi_{t}^{3}:=\mathrm{d} x^{3}+\mathrm{id} x^{6}
\end{array} ;\right.
$$

therefore

$$
\left\{\begin{array}{l}
\mathrm{d} \varphi_{t}^{1}=\mathrm{i} d \alpha \wedge \mathrm{d} x^{4} \\
\mathrm{~d} \varphi_{t}^{2}=0 \\
\mathrm{~d} \varphi_{t}^{3}=0
\end{array} ;\right.
$$

note that $\ell=\ell\left(x^{1}, x^{4}\right)$ gives rise to integrable almost-complex structures, in fact to Kähler structures; therefore, in such a case $J_{t, \ell}$ is $\mathcal{C}^{\infty}$-pure-and-full. Again, we assume $\ell=\ell\left(x^{3}\right)$ not constant. The $J_{t, \ell \text {-anti-invariant real closed }}$ 
2 -forms are

$$
\begin{aligned}
\psi= & \frac{C}{\alpha}\left(\mathrm{d} x^{13}-\alpha \mathrm{d} x^{46}\right)+D\left(\mathrm{~d} x^{16}-\alpha \mathrm{d} x^{34}\right)+E\left(\mathrm{~d} x^{23}-\mathrm{d} x^{56}\right) \\
& +F\left(\mathrm{~d} x^{26}-\mathrm{d} x^{35}\right)
\end{aligned}
$$

where $C, D, E, F \in \mathbb{R}$.

So, for small $t \neq 0$, we have the upper-semicontinuity property

$$
\operatorname{dim}_{\mathbb{R}} H_{J_{t, \ell}}^{(2,0),(0,2)}\left(\mathbb{T}_{\mathbb{C}}^{3}\right)_{\mathbb{R}} \leq 4<6=\operatorname{dim}_{\mathbb{R}} H_{J}^{(2,0),(0,2)}\left(\mathbb{T}_{\mathbb{C}}^{3}\right)_{\mathbb{R}}
$$

Unfortunately, the explicit computation of $H_{J_{t, \ell}}^{(1,1)}\left(\mathbb{T}_{\mathbb{C}}^{3}\right)_{\mathbb{R}}$ seems to be not so simple. In particular, it is not clear if $J_{t, \ell}$ remains $\mathcal{C}^{\infty}$-full (note that $J_{t, \ell}$ is $\mathcal{C}^{\infty}$-pure by [13, Proposition 2.7], see also [20, Proposition 3.2]).

Now, we recall how to construct curves of almost-complex structure via a $J$-anti-invariant real 2-form, as in [23].

Let $(M, J)$ be an almost-complex manifold; take $g$ a $J$-Hermitian metric and $\gamma$ a real 2-form in $\wedge^{2,0} M \oplus \wedge^{0,2} M$. Define $V$ to be the endomorphism of the tangent bundle such that

$$
\gamma(\cdot, \cdot)=: g(V \cdot, \cdot)
$$

a direct computation shows that $V J+J V=0$. Therefore, setting

$$
L \stackrel{\text { def }}{=} \frac{1}{2} V J
$$

we have $L J+J L=0$. At this point, for small $t$, define $J_{t, \gamma}$ as in (4.2):

$$
J_{t, \gamma} \stackrel{\text { def }}{=}(\mathrm{id}-t L) J(\mathrm{id}-t L)^{-1} \text {; }
$$

therefore, $\left\{J_{t, \gamma}\right\}_{t \in(-\varepsilon, \varepsilon)}$ is a curve of almost-complex structures naturally associated with $\gamma$.

We give an example of a $\mathcal{C}^{\infty}$-pure-and-full structure on a non-Kähler manifold such that the stability property holds along a curve constructed in such a way. Let

$$
\operatorname{Sol}(3) \stackrel{\text { def }}{=}\left\{\left(\begin{array}{cccc}
\mathrm{e}^{c z} & 0 & 0 & x \\
0 & \mathrm{e}^{-c z} & 0 & y \\
0 & 0 & 1 & z \\
0 & 0 & 0 & 1
\end{array}\right) \in \operatorname{GL}(4 ; \mathbb{R}) \mid x, y, z \in \mathbb{R}\right\} .
$$

Then $\operatorname{Sol}(3)$ is a completely solvable Lie group. For a suitable $c \in \mathbb{R}$, there exists a cocompact discrete subgroup $\Gamma(c)$ such that

$$
M \stackrel{\text { def }}{=} \Gamma(c) \backslash \operatorname{Sol}(3)
$$

is a compact three-dimensional solvmanifold. Define

$$
N^{6}(c) \stackrel{\text { def }}{=} M \times M .
$$


The manifold $N^{6}(c)$ has been studied in [7] as an example of a cohomologically Kähler manifold; M. Fernández et al. proved in [17] that it has no Kähler structures, although it is formal and it has a symplectic structure satisfying (HLC). In $[\mathbf{2 0}]$ a family of $\mathcal{C}^{\infty}$-pure-and-full structures on $N^{6}(c)$ is provided. Now, we will construct a curve of $\mathcal{C}^{\infty}$-pure-and-full almostcomplex structures on $N^{6}(c)$.

Let $\left\{e^{i}\right\}_{i \in\{1, \ldots, 6\}}$ be a coframe for $N^{6}(c)$; the structure equations are

$$
\left\{\begin{array}{l}
\mathrm{d} e^{1}=c e^{1} \wedge e^{3} \\
\mathrm{~d} e^{2}=-c e^{2} \wedge e^{3} \\
\mathrm{~d} e^{3}=0 \\
\mathrm{~d} e^{4}=c e^{4} \wedge e^{6} \\
\mathrm{~d} e^{5}=-c e^{5} \wedge e^{6} \\
\mathrm{~d} e^{6}=0
\end{array}\right.
$$

Let $J$ be the almost-complex structure given by

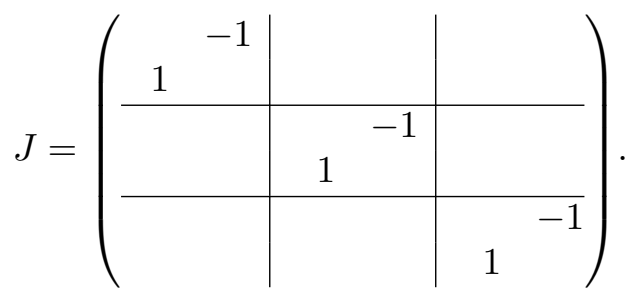

By Hattori-Nomizu theorem one computes immediately

$$
H_{d R}^{2}\left(N^{6}(c) ; \mathbb{R}\right)=\operatorname{span}_{\mathbb{R}}\left\{e^{1} \wedge e^{2}, e^{3} \wedge e^{6}-e^{4} \wedge e^{5}, e^{3} \wedge e^{6}+e^{4} \wedge e^{5}\right\} ;
$$

hence $N^{6}(c)$ is a $\mathcal{C}^{\infty}$-pure-and-full and pure-and-full manifold, the harmonic representatives being of pure degree. Note that

$$
H_{J}^{(2,0),(0,2)}\left(N^{6}(c)\right)_{\mathbb{R}}=\operatorname{span}_{\mathbb{R}}\left\{e^{3} \wedge e^{6}+e^{4} \wedge e^{5}\right\} .
$$

Put $\gamma:=e^{3} \wedge e^{6}+e^{4} \wedge e^{5}$; then the linear map $V$ representing $\gamma$ as in (4.4) is

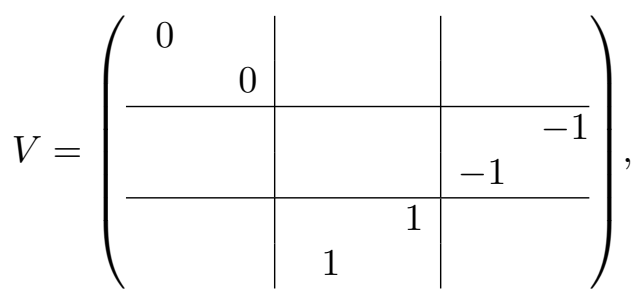


and then it is immediate to compute

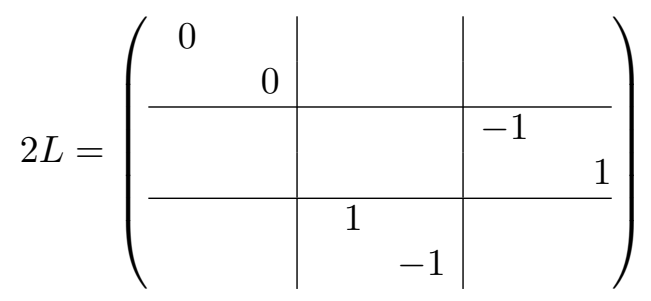

and

Set

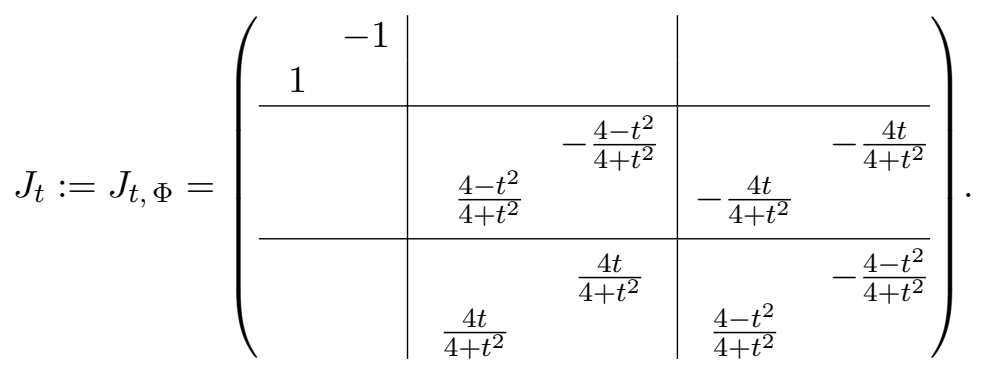

$$
\alpha:=: \alpha(t) \stackrel{\text { def }}{=} \frac{4-t^{2}}{4+t^{2}}, \quad \beta:=: \beta(t) \stackrel{\text { def }}{=} \frac{4 t}{4+t^{2}} .
$$

A coframe for the $J_{t}$-holomorphic cotangent bundle is given by

$$
\left\{\begin{array}{l}
\varphi_{t}^{1}=e^{1}+\mathrm{i} e^{2} \\
\varphi_{t}^{2}=e^{3}+\mathrm{i}\left(\alpha e^{4}+\beta e^{6}\right) \\
\varphi_{t}^{3}=e^{5}+\mathrm{i}\left(-\beta e^{4}+\alpha e^{6}\right)
\end{array} .\right.
$$

The closed 2-forms

$$
\frac{1}{2 \mathrm{i}} \varphi_{t}^{1 \overline{1}}, \quad \frac{1}{2 \mathrm{i}} \varphi_{t}^{3 \overline{3}}-\frac{\alpha}{c} \mathrm{~d} e^{5}, \quad \frac{1}{2 \mathrm{i}}\left(\beta \varphi_{t}^{2 \overline{2}}+\alpha\left(\varphi_{t}^{2 \overline{3}}-\varphi_{t}^{\overline{2} 3}\right)\right)+\frac{1}{2 \mathrm{i}} \varphi_{t}^{3 \overline{3}}
$$

generates three different cohomology classes; hence, for small $t \neq 0$, we get

$$
H_{d R}^{2}\left(N^{6}(c) ; \mathbb{R}\right)=H_{J_{t}}^{(1,1)}\left(N^{6}(c)\right)_{\mathbb{R}} ;
$$

this implies that $J$ is $\mathcal{C}^{\infty}$-full as well as pure. A straightforward computation yields

$$
\begin{aligned}
& H_{d R}^{4}\left(N^{6}(c) ; \mathbb{R}\right)= \operatorname{span}_{\mathbb{R}}\left\{*_{g}\left(\frac{1}{2 \mathrm{i}} \varphi_{t}^{1 \overline{1}}\right), *_{g}\left(\varphi_{t}^{3 \overline{3}}-\frac{\alpha}{c} \mathrm{~d} e^{5}\right)+\frac{\alpha}{c} \mathrm{~d}\left(e^{125}\right),\right. \\
&\left.\frac{\alpha}{4}\left(\varphi_{t}^{12 \overline{1} \overline{3}}+\varphi_{t}^{\overline{1} \overline{2} 13}\right)+\frac{\beta}{4} \varphi_{t}^{12 \overline{1} \overline{2}}+\frac{\alpha \beta}{c} \mathrm{~d}\left(e^{125}\right)\right\} \\
&=H_{J_{t}}^{(2,2)}\left(N^{6}(c)\right)_{\mathbb{R}} .
\end{aligned}
$$

Therefore, $N^{6}(c)$ is also $\mathcal{C}^{\infty}$-full at the fourth stage and, consequently, it is full as well as $\mathcal{C}^{\infty}$-pure.

Summarizing, we have proved the following. 
Theorem 4.1. There exists a compact manifold $N^{6}(c)$ such that:

(i) $N^{6}(c)$ admits a $\mathcal{C}^{\infty}$-pure-and-full almost-complex structure $J$;

(ii) each harmonic form of type $(2,0)+(0,2)$ gives rise to a curve $\left\{J_{t}\right\}_{t \in(-\varepsilon, \varepsilon)}$ of $\mathcal{C}^{\infty}$-pure-and-full almost-complex structures on $N^{6}(c)$;

(iii) furthermore, the map

$$
t \mapsto \operatorname{dim}_{\mathbb{R}} H_{J_{t}}^{(2,0),(0,2)}\left(N^{6}(c)\right)_{\mathbb{R}}
$$

is an upper-semicontinuous function at $t=0$.

\section{References}

[1] L. Alessandrini and M. Andreatta, Closed transverse ( $p, p)$-forms on compact complex manifolds, Compos. Math. 61(2) (1987), 181-200.

[2] L. Alessandrini and G. Bassanelli, Small deformations of a class of compact nonKähler manifolds, Proc. Amer. Math. Soc. 109(4) (1990), 1059-1062.

[3] M. Audin and J. Lafontaine (eds.), Holomorphic curves in symplectic geometry, in Progress in Mathematics, Vol. 117, Birkhäuser Verlag, Basel, 1994.

[4] W. P. Barth, K. Hulek, C. A. M. Peters and A. Van de Ven, Compact complex surfaces, 2nd ed., Ergebnisse der Mathematik und ihrer Grenzgebiete. 3. Folge. A Series of Modern Surveys in Mathematics (Results in Mathematics and Related Areas. 3rd Series. A Series of Modern Surveys in Mathematics), Vol. 4, Springer-Verlag, Berlin, 2004 .

[5] F. A. Belgun, On the metric structure of non-Kähler complex surfaces, Math. Ann. $\mathbf{3 1 7}(1)$ (2000), 1-40.

[6] C. Benson and C. S. Gordon, Kähler and symplectic structures on nilmanifolds, Topology 27(4) (1988), 513-518.

[7] C. Benson and C. S. Gordon, Kähler structures on compact solvmanifolds, Proc. Amer. Math. Soc. 108(4) (1990), 971-980.

[8] S. Console and A. Fino, Dolbeault cohomology of compact nilmanifolds, Transform. Groups 6(2) (2001), 111-124.

[9] G. de Rham, Differentiable manifolds, Grundlehren der Mathematischen Wissenschaften [Fundamental Principles of Mathematical Sciences], Vol. 266, SpringerVerlag, Berlin, 1984, Forms, currents, harmonic forms, Translated from the French by F. R. Smith, With an introduction by S. S. Chern.

[10] P. Deligne, P. Griffiths, J. Morgan and D. Sullivan, Real homotopy theory of Kähler manifolds, Invent. Math. 29(3) (1975), 245-274.

[11] J.-P. Demailly, Complex analytic and differential geometry, 2007, available at http://www-fourier.ujf-grenoble.fr/ demailly/manuscripts/agbook.pdf.

[12] S. K. Donaldson, Two-forms on four-manifolds and elliptic equations, Inspired by S. S. Chern, Nankai Tracts Math., Vol. 11, World Sci. Publ., Hackensack, NJ, 2006, pp. $153-172$.

[13] T. Draghici, T.-J. Li and W. Zhang, Symplectic forms and cohomology decomposition of almost complex four-manifolds, Int. Math. Res. Not. IMRN 2010(1) (2010), 1-17.

[14] T. Draghici, T.-J. Li and W. Zhang, On the J-anti-invariant cohomology of almost complex 4-manifolds, arXiv:1104.2511v1 [math.SG] . 
[15] M. Fernández and A. Gray, The Iwasawa manifold, Differential Geom., Peñíscola 1985, Lecture Notes in Math., 1209, Springer, Berlin, 1986, 157-159.

[16] M. Fernández, M. de León and M. Saralegui, A six dimensional compact symplectic solvmanifold without Kähler structures, Osaka J. Math 33(1) (1996), 19-34.

[17] M. Fernández, V. Muñoz and J. A. Santisteban, Cohomologically Kähler manifolds with no Kähler metrics, Int. J. Math. Math. Sci. 2003(52) (2003), 3315-3325.

[18] A. Fino and G. Grantcharov, On some properties of the manifolds with skewsymmetric torsion, Adv. Math. 189(2) (2004), 439-450.

[19] A. Fino, M. Parton and S. M. Salamon, Families of strong KT structures in six dimensions, Comment. Math. Helv. 79(2) (2004), 317-340.

[20] A. Fino and A. Tomassini, On some cohomological properties of almost complex manifolds, J. Geom. Anal. 20(1) (2010), 107-131.

[21] A. Hattori, Spectral sequence in the de Rham cohomology of fibre bundles, J. Fac. Sci. Univ. Tokyo Sect. I 8(1960) (1960), 289-331.

[22] K. Kodaira and D. C. Spencer, On deformations of complex analytic structures. III. Stability theorems for complex structures, Ann. Math. 71(2) (1960), 43-76.

[23] J. Lee, Family Gromov-Witten invariants for Kähler surfaces, Duke Math. J. 123(1) (2004), 209-233.

[24] T.-J. Li and W. Zhang, Comparing tamed and compatible symplectic cones and cohomological properties of almost complex manifolds, Comm. Anal. Geom. 17(4) (2009), 651-684.

[25] J. Milnor, Curvature of left-invariant metrics on Lie groups, Adv. Math. 21(3) (1976), 293-329.

[26] I. Nakamura, Complex parallelisable manifolds and their small deformations, J. Differential Geometry 10 (1975), 85-112.

[27] K. Nomizu, On the cohomology of compact homogeneous spaces of nilpotent Lie groups, Ann. Math. 59(2) (1954), 531-538.

[28] Y. Sakane, On compact complex parallelisable solvmanifolds, Osaka J. Math. 13(1) (1976), 187-212.

[29] J. Streets and G. Tian, A parabolic flow of pluriclosed metrics, Int. Math. Res. Not. IMRN 2010(16) (2010), 3101-3133, arXiv:0903.4418 [math.DG].

Dipartimento di Matematica "L. Tonelli"

UNIVERSITÀ DI PISA

Largo Bruno Pontecorvo 5, 56127

Pisa, ItALY

Dipartimento di Matematica

Università di PARMA

Parco Area delle Scienze 53/A, 43124

PARMA, ITALY

E-mail address: angella@mail.dm.unipi.it

E-mail address: adriano.tomassini@unipr.it

Received 06/01/2010, accepted 06/02/2010 
We would like to thank Tedi Draghici, Tian-Jun Li and Weiyi Zhang for their very useful comments and for pointing us the reference [14]. We are also pleased to thank the anonymous referee for fruitful suggestions and remarks for a better presentation of the results. This work has been originally developed as partial fulfillment for the first author's Master Degree in Matematica Pura e Applicata at Università di Parma under the supervision of the second author. This work was supported by the Project MIUR "Geometric Properties of Real and Complex Manifolds" and by GNSAGA of INdAM. 